\title{
Medizin in (Latein)Amerika vor der Conquista Teil l: Allgemeines und Andenregion
}

\author{
Reto Brignoli1, Reinhard Saller2 \\ 1 Facharzt für klinische Pharmakologie, CH-Rueschlikon; 2 UniversitätsSpital, Institut für Naturheilkunde, CH-Zürich
}

$\mathrm{M}$ it verschiedenen Beweggründen haben sich in den letzten Jahren zahlreiche Autoren mit der traditionellen Medizin unterschiedlichster Völker befasst [Vergl. 1,2]; sie könnte auch zur Entwicklung neuer Wirkstoffe beitragen [3]. Nachstehender ,Essay' soll dem Leser in einigen Pinselstrichen wie ein Aquarell - eine Idee über die Ursprünge der Volksmedizin in (Latein)Amerika vermitteln. Durch einen Ausflug in die Zeit vor der Conquista (d.h. der Eroberung durch die Europäer) werden im Teil I allgemeine Aspekte und die Medizin in der Andenregion behandelt werden. Teil II behandelt die Medizin in den Mesoamerikanischen Kulturen.

Wer sich heute mit der Volksmedizin in (Latein)Amerika befassen will, wird - mit Ausnahme von einigen Randregionen - ein Gemisch von verschiedenen Traditionen antreffen. Amerika wurde durch die Europäer militärisch, politisch, kulturell und religiös erobert und da, wo nicht genügend „Indios ${ }^{1 \text { “ }}$ die harte Arbeit und die eingeschleppten Epidemien überlebten, mit Sklaven aus Schwarzafrika besiedelt. Besonders gravierend wirkten sich die Pocken aus (von den Azteken Hueyzáhuatl oder Hueycocoliztli genannt und sicher ein bedeutender Alliierter von Hernán Cortés bei der Eroberung Mexikos).

Die Art der Eroberung prägt das heutige ethnisch-kulturelle Bild: Während in Nordamerika die Besiedlung durch protestantische Familien vorwiegend europäische Einflüsse mit sich brachte, wurden Zentral- und Südamerika militärisch erobert, wobei

1 Bewohner der 'Occidentali India'
Nachstehender ,Essay' soll dem Leser eine Idee über die Ursprünge der Volksmedizin in (Latein)Amerika vermitteln. Amerika wurde durch die Europäer erobert und da, wo nicht genügend Einheimische die harte Arbeit und die eingeschleppten Epidemien überlebten, mit Sklaven aus Schwarzafrika besiedelt. Die heutigen Volksglauben stellen einen Synkretismus aus althergebrachten Göttern und dem europäischem (katholischen) Christentum dar. Die "echte" althergebrachte Volksmedizin in (Latein)Amerika ist heute kaum mehr anzutreffen. Das Wissen wurde durch direktes Training weiter gegeben, es gab keine schriftlichen Dokumente. Man ist deshalb auf Exkursionen zu den Hauptkulturzentren zur Zeit der Eroberung angewiesen. Die Zeit der Eroberung ist eine Periode der Globalisierung, auch was infektiöse Krankheiten betrifft (Pocken, Masern, Cholera, usw.); gewisse Pflanzen gelangten nicht nur nach Europa sondern auch nach Afrika. Paleopathologische Studien zeigen, dass die Gesundheit der Einheimischen nicht besser als die der europäischen Zeitgenossen war; die mittlere Lebenserwartung wird auf ca. 38-43 Jahre geschätzt. Wie am Beispiel der Andenkulturen gezeigt wird, trifft man einerseits auf eine interpretationsfreie Empirie, andrerseits auf animistische Vorstellungen der Krankheiten (bez. Andenregion z.T. beschrieben in den "Comentarios Reales"). Krankheiten entstehen als Folge eines Ungleichgewichts zwischen Körper, Geist(er) und Göttern. Halluzinogene gehörten zum zentralen Armamentarium der Schamanenmedizin; grösste Bedeutung kam in den Andenregionen der Coca zu, nebst zahlreichen anderen Pflanzen. Die anthropomorphen ,Huaco' Vasen der Mochicas waren das Werk von Frauen und vermitteln auch die Gesundheitsvorstellungen und Erfahrungen derselben; die Thematik steht oft in scharfem Kontrast zu den patriarchalisch geprägten Denkmälern.

Schlüsselwörter: Arnzeipflanzen, Ethnobotanik, traditionelle Medizin, Paleopathologie, Inka, Mochica

\section{Precolumbian Medicine in (Latin)America Part I: General Aspects and Andean Region}

This ,Essay' provides an overview of the origins of popular (traditional) medicine in (Latin) America. The Americas were conquered by the Europeans and, wherever the number of aborigines surviving hard labour and imported epidemics declined, they were replaced by slaves from black Africa. Popular beliefs nowadays represent an amalgamation of the former deities and the European (catholic) Christian beliefs. It is almost impossible to find places where 'traditional' popular medicine is still practiced. Traditions were transmitted through training since there were no written documents. It is therefore necessary to reach back to the time of the conquest at the latest. The time of the conquest also was also one of globalisation, including infectious diseases (smallpox, measles, cholera, etc.) and plants which reached Europe and Africa. Paleopathological studies show that health among aborigines was no better than among European counterparts; mean life expectancy is estimated at around 38-43 years. Taking the cultures of the Andean regions as an example, one can show the coexistence of medical empiricism with animistic conceptions of disease (described to some extent in the "Comentarios Reales" for the Andean region). Diseases were considered the consequence of a lack of balance between body, spirit(s) and gods. Hallucinogens played a central role in the medicine of the shamans; most important was the coca-leaf in the Andean regions, among the panoply of herbal medicines. The anthropomorphic vases of the Mochica culture (,Huaco' Vases) were produced by women and also reflect their health-related views and experiences; the themes dealt with are in sharp contrast to those found in the monuments, with their patriarchal imprinting.

Key Words: medicinal plants, ethnobotany, traditional medicine, Paleopathology, Incas, Mochicas 
sich die „Conquistadores“ häufig indianische Frauen nahmen. Dies führte dazu, dass in diesen Indio/Mestizenregionen die Einflüsse der Urbevölkerung auch heute noch eindeutig sind. Die „Integration“ dieser Urbevölkerung war besonders erfolgreich in den kulturellen Hochburgen Zentralamerikas und der Andenregion; in Bolivien, Ecuador, Guatemala und Peru leben heute über $40 \%$ Indios [2]. Wichtig im $\mathrm{Zu}$ sammenhang mit den Indios ist, dass diesen, im Gegensatz zu Schwarz-Afrikanern, eine Seele zuerkannt wurde (Paulus III, Bula Sublimis Deus - 1537) was einerseits die Conquista „rechtfertigte“, zugleich aber auch den Conquistadores seelisch-moralische Obligationen auferlegte (abgesehen von der Ausbeutung und dem Raubbau, um die Habsburgerkriege zu finanzieren).

Die heutigen Volksglauben und Vorstellungen stellen einen Synkretismus aus althergebrachten Göttern und Machtstrukturen einerseits, und dem europäischen katholischen Christentum andererseits dar. Derartige Fusionen wurden zum Teil ,ex professo' gefördert (Jesuiten). Beispiele hierfür sind heute der ,Pacha Mama' Kult im Inkareich oder die sehr aktuellen Macumba, Candomblé usw. im afrikanisch geprägten Atlantik/Karibik-Raum. Für die Inka war Pachamama die Göttin Mutter $($ Pach $a=$ Erde $)$ und somit die Fruchtbarkeitsgöttin, verheiratet mit Inti, dem Sonnengott [4]. Sie wird heute wieder zunehmend - vermischt mit der Gestalt der Gottesmutter Maria - in vielen Gegenden Perus, Boliviens, Ecuadors, Argentiniens und in Chile verehrt. Zum Teil wurden auch Feste umfunktioniert, so feiern die Aymará der Andenregion hauptsächlich Karneval (mara anata) als Fruchtbarkeitsfest, während der Weihnacht kaum Bedeutung beigemessen wird.

Nach der Eroberung entstanden auch neue medizinische Konzepte oder Diagnosen, deren Wurzeln nicht immer klar sind. So sind gewisse Volksmedizinische Begriffe wie z.B. der „Empacho ${ }^{2 “}$ - eine vorwiegend Kinder betreffende

\footnotetext{
2 empacho = Magenverstimmung = Verlegenheit; von verb. empachar $=$ hindern $=$ überladen $=$ ver derben = behindern; verlegen werden. Stammt angeblich vom Franz, 'empêcher' bzw. von Altoccitanischen 'empachar'
}

Verdaungsstörung - auch heute im gesamten lateinamerikanischen Bereich bekannt, nicht aber in Europa [5].

Demzufolge ist die „echte“ althergebrachte Volksmedizin in (Latein)Amerika heute nur bei gewissen Stämmen des Amazonas oder Orinoco's anzutreffen, ansonsten ist man auf die überlieferten Dokumente aus der Zeit der Conquista angewiesen. Die Schamanen-Medizin hat zwangsläufig ihr mystisch-kulturelles Umfeld verloren, und ist häufig Opfer von Scharlatanen geworden; traditionelle und moderne Medizin koexistieren mit grosser Mühe und wenig Verständnis für einander [6]. Die traditionelle Medizin wird heute von „Curanderos“, die Geburtshilfe von „Comadronas“ praktiziert, diese werden in den armen Andenländern auch offiziell anerkannt [2]. Um eine unvoreingenommene Idee $\mathrm{zu}$ bekommen, was 'echtes' medizinisches Wissen der Indios war, bieten sich Exkursionen zu den Hauptkulturzentren zur Zeit der Eroberung an.

\section{Die Folgen der ,Conquista': Globalisierung}

Die Zeit der Eroberung stellt eine Periode der Globalisierung dar, auch was infektiöse Krankheiten betrifft (Pocken, Masern, Cholera und die Pest; Syphilis aus Amerika?, usw.). Allerdings glückte der „Technologie-Transfer“ nie eins zu eins - durch Missverständnisse, Vorurteile und Widerstand gegen das Neue - wie das Beispiel der Kartoffel zeigt. Das Überleben hat die Kartoffel im rauen Herzen der schroffen Berge der Anden gelernt. Die Kultivierung der Knolle in vielen hundert Varietäten (ca. 600) ist dort bis 7000 Jahre v. Chr. belegt. Die Indios stellten zur Haltbarmachung bereits früh eine Art Trockenkonserve her, die sie «Chuño» nannten. Die Knolle selbst nannten sie «Papa». Die Spanier brachten die Kartoffel als bereits alte Kulturpflanze in der Folgezeit (letztes Viertel des XVI. Jhs.) vom Hochland des heutigen Perus und Boliviens an den Hof von Spanien, worauf in der Nähe von Sevilla erste Pflanzungen angelegt wurden. Die Pflanze hatte aber noch 100 Jahre nach ihrer Entdeckung mit vielen Vorurteilen zu kämpfen. Man glaubte, sie verursache Aussatz, Lungenschwindsucht und Rachitis. Die Kartoffel wurde deshalb ursprünglich wegen ihrer Blüten auch eher als botanische Kuriosität und Zierpflanze, denn als Nutzpflanze verstanden. Viele grosse Männer mussten sich zuerst dafür einsetzen, dass die Kartoffel auch essbar ist. Philipp II von Spanien (1527-1598) überreichte dem Papst in Rom die ersten Kartoffelpflanzen noch als königliches Geschenk [7].

Einige Pflanzen gelangten nicht nur nach Europa, sondern auch nach Afrika und Asien, wo sie rasch als Nahrungsund als Heil- bzw. Medizinalpflanzen Bedeutung erreichten. So z.B. in Mozambique [8], wo Avocados (Persea americana Miller), Paprika, (Capsicum annum L., Capsicum frutescens L.), Caju (Anacardium occidentale L.), Guayaba (Psidium guayaba L.), Papaya (Carica papaya L.), Tomate (Lycopersicon esculentum Mill.), Tuna (Opuntia ficusindica L.) und Mais (Zea mays L.) grosse Verbreitung erreichten. Die Anwendungen stimmten aber nicht unbedingt mit denen im Ursprungsland überein, wie Tabelle 1 an einigen Beispielen zeigt. Allerdings waren auch in den verschiedenen Regionen Amerikas die Anwendungen oft unterschiedlich.

Was trafen die Conquistadores an? Trotz des enormen technologischen Vorsprungs der Europäer empfanden die Conquistadores die lokale Medizin (und ihre Heilpflanzen) besser als die europäische. Bernardino de Sahagún beschreibt, dass Hernán Cortés in einem Brief an den, von schwerer Gicht geplagten [14], Kaiser Karl V diesen bat, keine Ärzte in das frisch eroberte Mexiko zu schicken, da die Mexikaner „die Erfinder der Medizin und die ersten Herbalmediziner (azt. Ticitl) seien“.

Paleopathologische Studien [15] zeigen jedoch, dass die Gesundheit der Indios im allgemeinen nicht besser als diejenige der Conquistadores war; die mittlere Lebenserwartung wird auf ca. 38-43 Jahre (Nasca, Peru) geschätzt [16]. $40 \%$ der Kinder starben in den ersten fünf Lebensjahren. Hunger meistens durch anhaltende Trockenperioden, Seuchen, wie Typhus, und Kriege sowie Komplikationen bei der Geburt bei Frauen und Unfälle bei den 
Tab. 1. Medizinische Anwendungen von einigen verbreiteten amerikanischen Pflanzen in der Andenregion und in Mozambique (aus [8])

\begin{tabular}{|c|c|c|c|c|c|}
\hline \multirow[t]{2}{*}{ Pflanze } & \multicolumn{3}{|c|}{ Amerika (Andenregion) } & \multicolumn{2}{|l|}{ Mozambique } \\
\hline & \multicolumn{2}{|c|}{ Pflanzenteil[e] } & Anwendungsbereich(e) & Pflanzenteil[e] & Anwendungsbereich(e) \\
\hline \multirow[t]{4}{*}{$\begin{array}{l}\text { Avocado } \\
\text { (Persea americana; *) }\end{array}$} & \multicolumn{2}{|c|}{$\begin{array}{l}\text { trockene oder frische } \\
\text { Blätter (Aufguss) \& } \\
\text { Samen (Aufguss) }\end{array}$} & $\begin{array}{l}\text { verdauungsfördernd, } \\
\text { Husten }\end{array}$ & \multirow{4}{*}{$\begin{array}{l}\text { trockene oder } \\
\text { frische Blätter } \\
\text { (Aufguss) }\end{array}$} & \multirow{4}{*}{$\begin{array}{l}\text { Blutarmut, Hypertonie, } \\
\text { u.a. }\end{array}$} \\
\hline & \multicolumn{2}{|c|}{$\begin{array}{l}\text { Blätter \& Rinde } \\
\text { (Aufguss) }\end{array}$} & $\begin{array}{l}\text { Nierenkoliken; } \\
\text { Infektionen der Harnwege }\end{array}$ & & \\
\hline & \multicolumn{2}{|c|}{$\begin{array}{l}\text { Fruchtschalen } \\
\text { (Ab- bzw. Auskochung), } \\
\text { Kerne (gerieben) }\end{array}$} & Wurmbefall & & \\
\hline & \multicolumn{2}{|c|}{$\begin{array}{l}\text { Kern (Ab- bzw. } \\
\text { Auskochung) }\end{array}$} & $\begin{array}{l}\text { Antiabortivum \& } \\
\text { Anticonceptivum }\end{array}$ & & \\
\hline \multirow[t]{4}{*}{$\begin{array}{l}\text { Guayaba } \\
(\text { Psidium guajava **) }\end{array}$} & \multicolumn{2}{|c|}{ Blätter (Aufguss) } & $\begin{array}{l}\text { Bauchschmerzen, } \\
\text { Diarrhöe }\end{array}$ & $\begin{array}{l}\text { Blätter A oder Wurzeln } \\
\text { (Aufguss) }\end{array}$ & Bauchschmerzen \\
\hline & \multicolumn{2}{|c|}{ Blätter (zerkaut) } & Zahnschmerzen & \multirow{3}{*}{ Blätter (Aufguss) } & \multirow{3}{*}{$\begin{array}{l}\text { Fieber, Husten, } \\
\text { Diabetes } \mathrm{m} \text {. }\end{array}$} \\
\hline & \multicolumn{2}{|c|}{ Blätter (Frischpflanzensaft) } & Augenlidentzündung & & \\
\hline & \multicolumn{2}{|l|}{ Frucht } & Diarrhöe, Wurmbefall & & \\
\hline \multirow[t]{2}{*}{$\begin{array}{l}\text { Paprika } \\
\text { (Capsicum annum } \\
\text { u.a. }{ }^{* *} \text { ) }\end{array}$} & \multicolumn{2}{|c|}{$\begin{array}{l}\text { Äusserlich / topisch: } \\
\text { Samen und } \\
\text { frische Blätter }\end{array}$} & $\begin{array}{l}\text { Analgesie, } \\
\text { (Zahnschmerzen) } \\
\text { Entzündungen, rheuma- } \\
\text { tische Beschwerden }\end{array}$ & frische Blätter (Saft) & Augenlidentzündung \\
\hline & \multicolumn{2}{|c|}{ Frucht/Samen, innerlich: } & $\begin{array}{l}\text { verdauungsfördernd, } \\
\text { Wurmbefall, } \\
\text { Hämorrhoiden }\end{array}$ & $\begin{array}{l}\text { Frische Blätter } \\
\text { (zerdrückt als } \\
\text { Suppositorium) }\end{array}$ & Hämorrhoiden \\
\hline \multirow{4}{*}{$\begin{array}{l}\text { Papaya } \\
\text { (Carica papaya L. }{ }^{* * *} \text { ) }\end{array}$} & \multirow{2}{*}{$\begin{array}{l}\text { Latex der } \\
\text { grünen } \\
\text { Frucht }\end{array}$} & innerlich: & Wurmbefall, Laxativum & \multirow{2}{*}{$\begin{array}{l}\text { grüne Frucht } \\
\text { (Ab- bzw.Auskochung) }\end{array}$} & \multirow{2}{*}{ Malaria } \\
\hline & & äusserlich: & Hautparasiten (Krätze) & & \\
\hline & \multicolumn{2}{|c|}{$\begin{array}{l}\text { Wurzel (Ab- bzw. } \\
\text { Auskochung) }\end{array}$} & $\begin{array}{l}\text { Wurmbefall, } \\
\text { Harnwegsinfekte }\end{array}$ & Wurzel (Mazeration) & $\begin{array}{l}\text { Bauchschmerzen, } \\
\text { Gonorrhöe }\end{array}$ \\
\hline & \multicolumn{2}{|l|}{ Blüten } & antikonzeptiv & $\begin{array}{l}\text { Frucht \& frische Samen } \\
\text { in Wasser }\end{array}$ & Laxativum; Wurmbefall \\
\hline \multicolumn{6}{|c|}{ A) auch gemeinsam mit den Blättern von Carica papaya angewendet. } \\
\hline \multicolumn{6}{|c|}{$\begin{array}{l}\text { *) 3-Oxoacyl-(acyl-carrier protein) reductase [9]; (2E,5E,12Z,15Z)-1-hydroxyheneicosa-2,5,12,15-tetraen-4-one, (2E,12Z,15Z)-1- } \\
\text { hydroxyheneicosa-2,12,15-trien-4-one, and (5E,12Z)-2-hydroxy-4-oxoheneicosa-5,12-dien-1-yl acetat und andere [10] }\end{array}$} \\
\hline \multicolumn{6}{|c|}{ **) Kein spezifischer identifiziert; reich an Quercetin. Pharmakologie beschrieben (u.a. [11]) } \\
\hline \multicolumn{6}{|c|}{ ***) Capsaicin Dihydrocapsaicin, Nordihydrocapsaicin, Homodihydrocapsaicin, Homocapsaicin³ } \\
\hline \multicolumn{6}{|c|}{ ****) Rohpapain / Papain [12,13] } \\
\hline
\end{tabular}

Männern verursachten die Grosszahl der Todesfälle. Derartige Studien zeigen auch, dass - besonders in Bevölkerungen, die vorwiegend Mais konsumierten, Eisenmangelanämien [17] recht häufig waren. Karies, Zahnverfärbun-

\footnotetext{
3 http://www.buetzer.info/fileadmin/pb/HTML-Files/ Capsaicin.htm

4 Für Zahlen gab es das Quipu oder Khipu, eine Knotenschrift der Inka, wobei an einem Hauptfaden Dutzende von Nebenfäden angeknüpft wurden, die mit verschiedenartigen Knoten versehen sind. Frühere Kulturen benützen eine einfachere Version, wobei keine Knoten geknüpft, sondern mit bunten Fäden farbige Umwicklungen vorgenommen wurden.
}

gen und andere odontologische Pathologien waren verbreitet, mit bedeutenden regionalen Unterschieden [18]. Auch Parasitosen, wie Ancylostoma duodenale [19], Trichinella spiralis, Cryptosporidium, Giardia und Cyclospora, wurden identifiziert [20]. Die Knochenpathologien sind naturgemäss - da dieses Material die Zeit gut überlebt sehr gut belegt [21,22] und zeigen in der Andenregion unter anderem Beispiele von ritueller Kopfdeformation, Arthrosen und Pott'scher Krankheit auf.

Allerdings gibt es nur sehr geringe direkte Überlieferungen der Urbevölkerung, da allgemein keine schriftlichen Dokumente - mit Ausnahme von zentralamerikanischen Hieroglyphen und Piktogrammen oder Khipu [23] in der Andenregion ${ }^{4}$ - vorhanden sind; das Wissen wurde durch direktes Training und mündliche Überlieferung weiter gegeben. Dementsprechend gibt es auch keine direkten Angaben die Medizin betreffend. Allerdings haben verschiedene Autoren (vorwiegend katholische Geistliche) die Erzählungen, Sitten und Gebräuche in der Zeit vor 


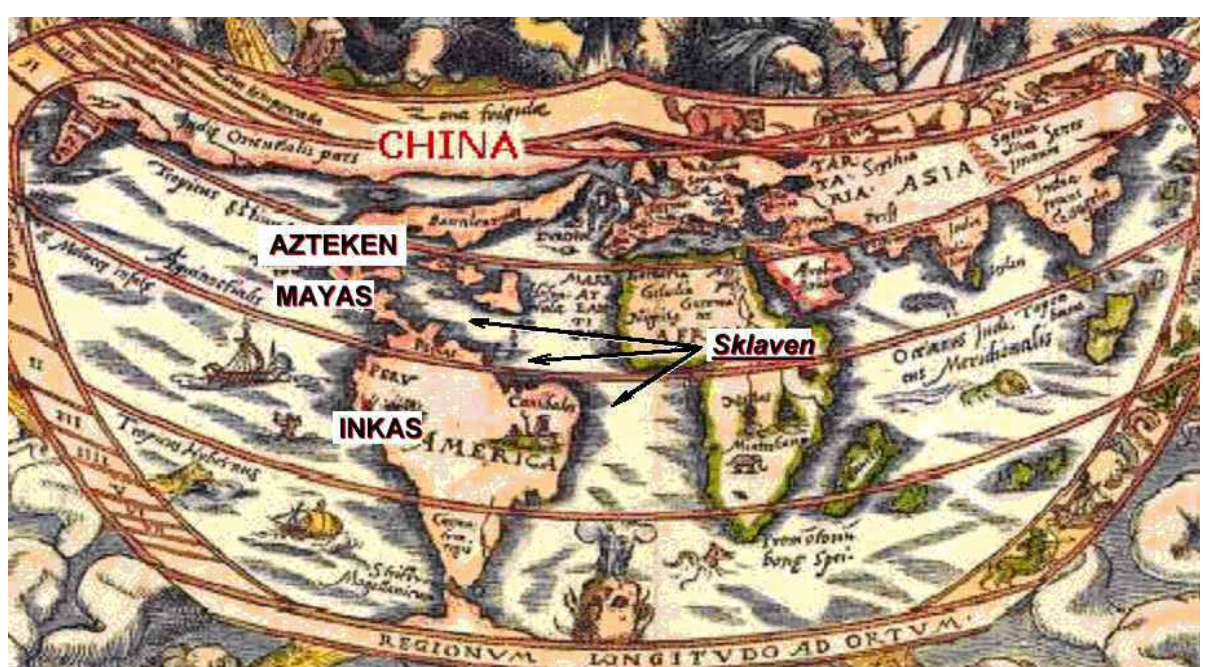

Abb. 1. Kulturelle Hochburgen zur Zeit der Conquista und Zustrom von Sklaven aus Schwarzafrika.

der Eroberung in den verschiedenen kulturellen ,Hochburgen' der Mayas, Azteken und Inkas festgehalten [24] (vergl. Abb. 1). Martín de la Cruz, ein durch Priester in Tlatelolco ausgebildeter mexikanischer „Indio“, publizierte 1552 das wahrscheinlich erste illustrierte spezifische Medizinalbuch der neuen Welt, das „Libellus de Medicinalibus Indorum Herbis“, bekannter als "Códice Badiano” (in Latein und Nahuatl verfasst). Im Andengebiet wurden die indianischen Traditionen, inklusive der Medizin, durch verschiedene Autoren kurz nach der Conquista umfangreich dokumentiert: Autoren wie Diego de Castro, Titu Cusi Yupanqui, Juan de Santa Cruz, Pachacuti Yamqui Salcamaygua, Felipe Huamán

\footnotetext{
5 siehe auch http://www.google.com/intl/qu/ auf Quechua und Microsoft Quechua für Office 2003 [LIP.EXE]

6 As any other system of knowledge, traditional knowledge is embedded within specific worldviews. In this respect, modern science is not different, it is also anchored in a specific worldview and, more to the point, a specific view about man's relation to nature that is strongly instrumental. In contrast, the worldview embraced by traditional knowledge holders typically emphasizes the symbiotic nature of the relationship between humans and the natural world. Rather than opposing man and nature as in Occidental thought, traditional knowledge holders tend to view people, animals, plants and other elements of the universe as interconnected by a network of social relations and obligations. Holistic cosmologies that intertwine elements that are ecological and social, as well as empirical and spiritual, have confounded scientists who may seek to separate 'fact' from 'superstition'. Such a dualistic approach, however, contains certain dangers. Practices that appear in the first instance as superstitious to the outside observer may, once additional knowledge about the environment and culture is acquired, prove to be appropriate and empirically sound ways of coping with environmental problems.
}

Poma de Ayala; und ,last but not least' der Inca Garcilaso de la Vega schrieben zwar in Spanisch, sprachen und dachten aber in Quechua (Auch heute noch, nebst dem Aymará, die Lingua franca im Andengebiet ${ }^{5}$ ) [25].

Verschiedene Pflanzen mit pharmakologischer Wirkung wurden erst zur Zeit der „Conquista“ oder in der Gegenwart entdeckt. Beispiel hierfür ist das Chinin; dieses wurde von den Jesuiten um 1630 entdeckt und als fiebersenkendes Mittel während vieler Jahre von der Compañía de Jesús auch in Europa kommerzialisiert; seine Wirkung bei Malaria wurde erst im XIX Jahrhundert beschrieben. Ähnlich verhält es sich mit dem Curare, von den Indios wohl als Pfeilgift(e) benutzt, wobei die Natur der Wirkung der Curare-Gifte aus Strychnos (toxifera u.a.) und Chondrodendron (tomentosum u.a.) erst durch Claude Bernard (1813-1887) enträtselt wurde.

\section{Medizinische Vorstellungen der Urbevölkerung(en)}

Einerseits trifft man auf eine interpretationsfreie Empirie, die auf oralen Überlieferungen und ,Anlernen' von Jüngeren beruht, andrerseits auf vorwiegend animistische Vorstellungen des Lebens im Allgemeinen und der Krankheit im Speziellen an. Diese entstehen als Folge eines Ungleichgewichts zwischen Körper, Geist(ern) und Göttern.

So schreibt A. MENA [26], in der westlichen Welt unterscheiden wir zwischen Gesundsein und Gesundfühlen (idée de santé) bzw. zwischen Realität und Vorstellung der Realität. In den traditionellen östlichen Kulturen hingegen sind beide Dimensionen, das Natürliche und das Kulturelle, in eine Einheit integriert, ohne Notwendigkeit der Trennung verschiedener Gebiete. Gesundheit wird als ein Gleichgewicht von Energien verstanden (der Person und der verschiedenen Umfelder). In den traditionellen (i.B. mesoamerikanischen) Kulturen ist Gesundheit das Ergebnis eines dynamischen Gleichgewichts zwischen dem Menschen und dessen Anpassung an das soziale, natürliche, kulturelle und geistige Milieu, die ihrerseits auch interagieren. Von der allgemein akzeptierten Auffassung, dass die amerikanischen Völker asiatischen Ursprungs sind, könnte man diese Verwandtschaft auch - wie verschiedene Autoren suggerieren - auf das kulturelle Milieu und besonders auf das Verständnis von Krankheit/ Gesundheit ausdehnen. Ähnliche Konzepte beschreibt auch das International Council of Scientific Unions (ICSU) [27] bezüglich „traditional knowledge“, welches - wie ja auch das moderne wissenschaftliche Denken - in spezifischen Visionen der Welt verankert ist 6 .

\section{Psychodysleptika versus „alltägliche" Probleme}

Geist- oder bewusstseinsverändernde Eingriffe und Drogen hatten grosse Bedeutung, wohl als Konsequenz der vorwiegend ,holistischen' Vorstellungen. Auch die meisten der gut belegten Trepanationen [28] - wobei wohl zerkautes Coca als Lokalanästhetikum eingesetzt wurde - gehören höchstwahrscheinlich in diesen „medizinischen“ Bereich, obwohl auch posttraumatische Eingriffe belegt sind. Interessant ist, dass anfänglich ca. $30 \%$, zur Zeit der Inkas sogar ca. $70 \%$ der so operierten Patienten den Eingriff überlebten [29]. „Alltägliche“ Probleme hingegen, wie Geburten, Unfälle/Verletzungen, Hauterkrankungen usw. werden vom Volk (häufig Frauen!) betreut; dieses hat kaum Zugang zu der Schamanen-Medizin der Machthabenden, wobei die Übergänge fliessend sind.

Trance-Zustände mit Psychodysleptika [Halluzinogene] gehörten zum zen- 
tralen Armamentarium der Schamanenmedizin. Aus der Rinde des Yagé (Banisteriopsis caapi; eine Lianen-Art aus der Familie der Malpighiengewächse; enthält die Alkaloide Harmin, Harmalin, D-Tetrahydroharmin sowie N,N-Dimethyltryptamin) wird im Andenraum Ayahuasca mit anderen pflanzlichen Komponenten hergestellt ("vino de la muerte" d.h. Todeswein, wohl in Anspielung auf die Vorbereitung der geopferten Menschen). Das weitverbreitete Yopo ("tree-tobacco"; Piptadenia peregrina) enthält das Halluzinogen Bufotenin. Stimulierend wirken Pericá (virola colophila) und das Yoco (Paulinia yoco) sowie das verwandte Guaraná (Paulinia capana). Ebenfalls fanden die verschiedenen Datura-Arten verbreitet Anwendung, wobei die Tropanalkaloide Scopolamin und Hyoscamin die halluzinogenen Inhaltsstoffe dieser Pflanzen sind, i.B. im Andenraum Myaya (Datura ferox Linn.), in Zentralamerika Atlinan (aztek.) oder Toloatzin (Maya), (Torna loco, d.h. verrücktmachende Pflanze; Datura ceratocaula), etc.. In Zentralamerika wurden auch das psychedelisch wirksame Meskalin-haltige Peyotl (Lophophora williamsii), das Ergin-haltige Ololiuhqui (LSD-ähnliches Ergolin; Rivea corymbosa oder Turbina corymbosa) und Cochit-zapotl (von cochi = schlafen; Casimiroa edulis, Rutaceae) eingesetzt [30]. Für einigen Aufruhr sorgte kürzlich das Psilocybin (Psilocybe cubensis, Psilocybe semilanceata, u.a.) [31], das bei „mehr als sechzig Prozent der (36) Freiwilligen Effekte hatte, welche die Kriterien einer ,vollständigen mystischen Erfahrung' nach etablierten psychologischen Massstäben erfüllten“.

\section{Beispiel: Peru vor den Europäern}

\section{Schriftliche Überlieferungen}

Eine sehr umfangreiche schriftliche Überlieferung ist die vom Inca Garcilaso de la Vega (1539-1616), Sohn des Conquistador Garci Lasso de la Vega Vargas und der Chimpu Ocllo, getauft Isabel, Enkelin des Túpac Yupanqui und Cou-

\footnotetext{
7 http://www.ciedperu.org/productos/molle.htm

8 http://www.consilia-sa.ch/agsa/D/anhang_2_D.htm
}

sine von Atahualpa, dem letzten Inka.

Der Inca Garcilaso beschrieb in seinen „Comentarios Reales“ wohl die gängigsten Prozeduren, wie er sie in seiner Kindheit gesehen hat [32]. Dazu gehörten besonders der Aderlass und das Abführen ,mit kleinen weissen Knollen, die sowohl Durchfall und allgemeines Erbrechen verursachten'. Bei dieser Gelegenheit wurden auch Würmer und anderes Ungeziefer eliminiert. Der Aderlass erfolgte ,ohne grosse Kunst' jeweils mit einer Feuersteinlanzette in der Nähe der betroffenen bzw. schmerzhaften Stelle. Die Feuersteinlanzette wurde an einem Stäbchen festgemacht; durch dessen rasches Drehen erfolgte die Punktion der Ader schnell und schmerzlos. Sie massen der Körpertemperatur grosse Bedeutung bei, wobei der Schüttelfrost (Malaria) als „chucchu“ (Zittern, noch heute in der Umgangssprache gebräuchlich), und das Fieber als „Rupa“ (Verbrennen), bezeichnet wurden.

Unter den Pflanzen fand der „Mulli“ grosse Anwendung (Schinus molle Linn.), wobei die Blätter und Blüten für Umschläge gegen Gelenkschmerzen verwendet wurden (und werden 7 ), mit den Schalen der Früchten wurden/werden Getränke hergestellt. Maisgetränke (zara) waren sehr wirksam zur Behandlung von Nierenschmerzen und -steinen und wurden auch rasch von den Conquistadores adoptiert. Tabak (sairi) fand ebenfalls breite Anwendung; er wurde inhaliert um den „Kopf zu entleeren“. Auch tierische Produkte, wie Condorfedern (Vultur gryphus) oder Kolibris (Mettalura phoebe, Archilochus colubris u.a.) kamen zur Anwendung. Frauen fangen heute noch den Andenspecht (Colaptes rupicola; Quechua hak'akllu und pitu, u.a.; Aymara, yarakaka) oder sammeln dessen Federn; diese werden getrocknet und gemahlen eingenommen - häufig mit Fenchel gemischt (Foeniculum vulgare) - um die Milchproduktion der stillenden Mütter (und Haustiere) zu steigern [33]. Einzige Begründung ist, dass die Vorfahren (Quech. Machulas) diese Mittel schon benützt haben.

Grösste Bedeutung kam in den Andenregionen der Coca zu, wie auch die Haare von Mumien bezeugen [34] (Erythroxylum coca, Erythroxylum no- vogranatense u.a.). Die Blätter der Coca - die einem Schmetterling (Eloria noyesi) als Nahrung dienen, für die Pharaohameisen (Monomorium pharaonis) hingegen als Gift wirken - werden noch heute mit einer alkalischen Paste (llipta oder llucta) vermischt und in einer Backentasche gesaugt (chacchar oder acullicar). Allerdings enthalten die Cocablätter, nebst Cocain, zusätzliche Alkaloide mit grösstenteils noch unbekannten, wahrscheinlich modulierenden, Wirkungen. Die Hauptmetaboliten Benzoylecgonin und Ecgoninmethylester (Methylecgonin) sind auch in den Blättern anzutreffen. Sie entstehen durch enzymatische (Pseudocholinesterase) oder spontane Hydrolyse $^{8}$. Weitere Alkaloide der Blätter sind Cinnamoylcocain, Pseudotropine, Benzoyltropin, Tropacocain, alphaund beta-Truxillin, Hygrin, Cuscohygrin und Nicotin, u.a.. Der Inca Garcilaso beschrieb ausführlich die Anwendung der Coca durch die Einheimischen, um deren Ausdauer zu steigern und das Hungergefühl zu unterdrücken. Zudem sollte Coca auch zur Heilung verschiedenster Übel beitragen. Obwohl anfänglich von den Conquistadores verboten, da die Anwendung die Indios „Vom wahren Glauben ablenken würde“, schnellte der Gebrauch mit der Entdeckung der Silberminen in Potosí (4000m Höhe) massiv in die Höhe, z.B. auf 100.000 Cocakörbe im Jahre des Herrn 1548, mit der entsprechenden Einkommensverbesserung der Kirchenhierarchie welche die Plantagen kontrollierte. Da der Alkaloidgehalt der Cocablätter sehr rasch abnimmt wenn diese nicht ,lege artis' aufbewahrt werden, konnte man in Europa, während der Kolonialzeit, die Effekte der Coca nie nachvollziehen [35].

\section{Überlieferungen über darstellende Kunst; die Huacos}

Ähnlich wie im Mittelalter die biblische Geschichte in Bilderbuchformat in den Kirchen dargestellt wurde, wird von den Mochicas (400-1000 n.Chr.) und anderen Kulturen des Pazifischen Raumes das Wissen - ohne Vorhandensein einer Schrift - verbal und mittels den anthropomorphen Vasen vermittelt. Viele dieser Bräuche retteten sich von einer politischen Domination zur an- 
deren bis zum heutigen Tage. Die wohl grösste Sammlung von anthropomorphen Vasen findet man im Museum Larco Herrera ${ }^{9}$ in Lima, Perú, wo insgesamt 45’000 Vasen und Objekte chronologisch und thematisch klassifiziert ausgestellt werden (Nachstehend werden Elemente aus dieser Sammlung mit „Larco H. $\mathrm{N}^{\circ}$...“ gekennzeichnet). Weitere Huacos findet man ebenfalls im Museo Antropológico y Arqueológico in Lima, im Museo Arqueológico José Cassinelli, Trujillo, Perú, usw. [36].

Diese Vasen waren - wie auch die Weberei - das Werk von Frauen und vermitteln - zumindest teilweise - die Vorstellungen und Interessen derselben; die Darstellungen sind frisch und direkt und zeigen Alltägliches des gemeinen Volkes. Die Thematik steht in scharfem Kontrast zu den patriarchalisch geprägten Denkmälern, Palästen und Grabstätten, wie wir sie von den Grabstätten des Herrschers "Señor de Sipán” kennen (1987 entdeckt) ${ }^{10}$. So findet man sehr realistische Darstellungen von Tieren sowie der üblichen Nahrungsmittel wie der Mais (Zea mays) - wohl das Wichtigste; Bohnen (Phaseolus lunatus \& Phaseolus pallar), Kartoffeln (Solanum tuberosum), Yucas (Manihot esculenta), Chirimoyo (Anona chirimolia), verschiedene Kürbisarten (Cucurbita maxima \& Cucurbita pepo) etc., etc.

Dies betrifft auch die Medizin - oft unglaublich treffend dargestellt -, inklusive Geburtshilfe und Sexualkunde. Die Medizinmänner oder -frauen werden immer in prunkvoller Bekleidung mit Ohren und Nasenschmuck und mit ihrem einfachen Instrumentarium dargestellt; der Patient wurde jeweils im Liegen untersucht und die Götter um Rat angerufen [37].

\footnotetext{
9 Museo Larco Av. Bolívar 1515 - Pueblo Libre Lima 21 - Perú http://museolarco.perucultural.org.pe

10 Archäologen der Fundacion Wiese entdeckten 2005 die sehr gut erhaltene Priesterin(?) "Señora de Cao". Sie trug Tätowierungen mit Schlangen und Spinnen, die in der Andenregion die Fruchtbarkeit symbolisieren. Sie verstarb im Alter von etwa 25-30 Jahren.

${ }^{11}$ Auch Fetal-Face-Syndrom. Das Robinow-Syndrom ist ein Syndrom mit multiplen Fehlbildungen. Es wird durch rezessive Mutationen im ROR2-Gen verursacht. Die hauptsächlichen klinischen Symptome sind: sehr charakteristische faziale Dysmorphien (u.a. große, ausladende Stirn, flaches Gesichtsprofil, kurze Nase mit aufgeworfener Spitze, Hypertelorismus), mesomele Verkürzung der Arme, Brachydaktylie und hypoplastisches Genitale.
}

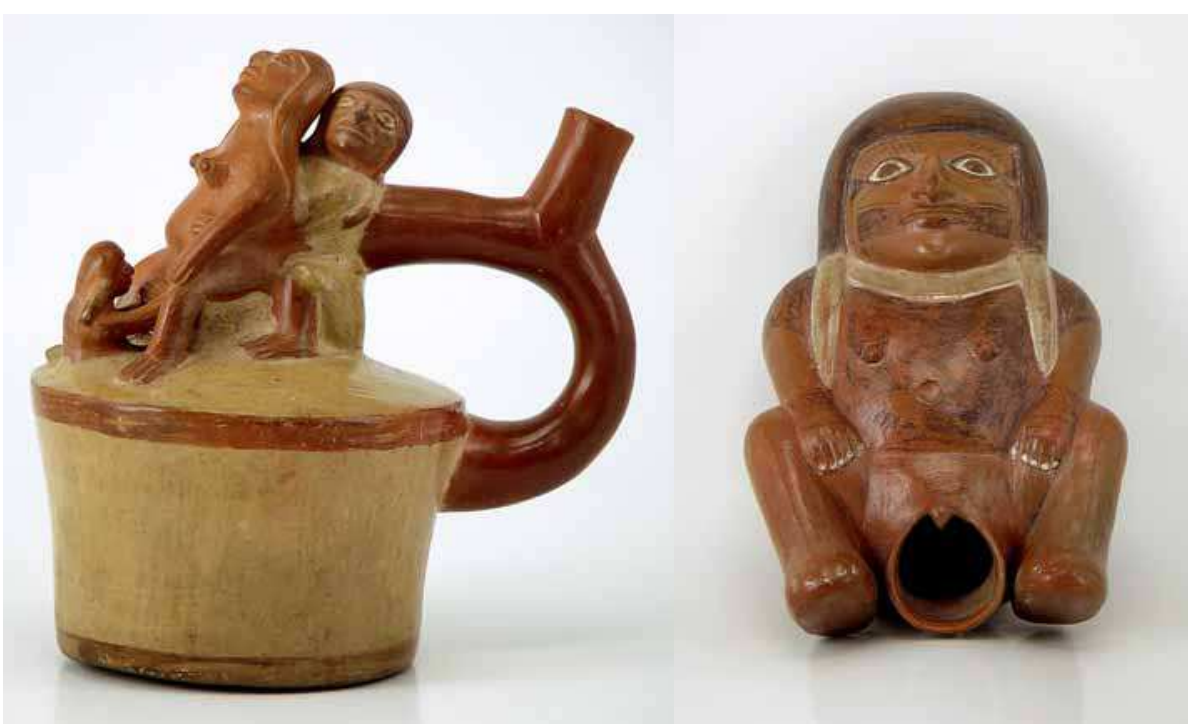

Abb. 2. Geburtshilfe (Larco H. N ML004424) - ein häufiges Thema - und Geburtskanal (Larco H. $N^{\circ}$ ML004426)

Die Geburten, jeweils in sitzender Position und mit mehreren HelferInnen, wurden sehr eindrücklich und wie in einem Instruktionsbilderbuch dargestellt (Larco H. $\mathrm{N}^{\circ}$ ML004424, Abbildung 2, wobei der Mann beim Pressen hilft, während die Hebamme das Kind in Empfang nimmt. Auch stillende Mütter sind ein häufiges Thema (Larco H. N ML012845 u.a.).

Ein wiederkehrendes Thema ist die Haut-Schleimhaut-Leishmaniase (Leishmania brasiliensis u.a.), die Übertragung erfolgt durch Sandfliegen Lutzomyia intermedius, Lu. Longipalpis, u.a.; (Larco H. N ML002641, ML002640 u.a.), d.h. die Uta, mit Geschwürsbildung, oder die Espundia, welche die von der Haut auf Rachen- und Mundschleimhaut übergreifende Form darstellt [38] und dort teilweise gefährliche chronische Wundinfektionen verursacht. Auch die Hypoplasie des Nasenflügels wird realitätsgetreu dargestellt [39].

Andere häufige Themen sind die manuelle Entlausung (Larco $\mathrm{H}$. $\mathrm{N}^{\circ} \mathrm{ML}$ 002306 u.a., siehe auch [40]), Cocakauende Menschen (Larco H. $\mathrm{N}^{\circ} \mathrm{ML}$ 002549 u.a.), Schilddrüsenerkrankungen wie Kropf (Larco H. N ML002724, ML002630 u.a.), Buckelige, Menschen mit Klumpfuss (Larco H.N ${ }^{\circ}$ ML002733), auch Blinde werden sehr eindrücklich dargestellt (Abb. 3-7) und sogar siamesische Zwillinge (Larco H. $\mathrm{N}^{\circ}$ ML00 2411). Abgesehen von den schon erwähnten Trepanationen sind ampu- tierte Gliedmassen mit einfachen Prothesen (Larco H. $\mathrm{N}^{\circ}$ ML002724 u.a.) recht häufig dargestellt und zeugen mit Beispielen der Stumpfvorbereitung vom chirurgischem Können der Mochicas, wie auch paleopathologische Funde belegen (Abb. 8) [41]. FacialisParesen und kongenitale Missbildungen wie Hasenscharte oder Robinow Syndrom ${ }^{11}$ wurden ebenfalls sehr treffend dargestellt [42].

Die Beschneidung scheint - anhand der Darstellung der männlichen Genitalien in Vasen- sehr verbreitet gewesen zu sein. Hierzu wurde wahrscheinlich das Tumi benutzt, ein breites Ritualmesser aus Kupfer in Halbkreisform mit senkrechtem Mittelgriff und der Darstellung eines Gottes oder eines Herrschers. Die Darstellungen verschiedenster Arten von sexuellen Handlungen - sowohl hetero- wie homosexuelle haben bei den Priestern der Conquistadores für gehörige Aufregung und heute für ebenso grosse Neugier bei den Touristen gesorgt. Was der eigentliche Zweck derartiger Darstellungen war, wie z.B. angeblich Analverkehr zur Empfängnisverhütung (Larco H. $\mathrm{N}^{\circ}$ ML004221 u.a.) oder die Fellatio (Larco H. N$^{\circ}$ ML004283 u.a.), wird uns wohl für immer verborgen bleiben.

Spätere Kulturen der Andenregionen haben vieles von den Mochicas übernommen und sicherlich auch weiterentwickelt, keiner zeigte aber wieder ihre Welt in solch unvoreingenommener Art. 


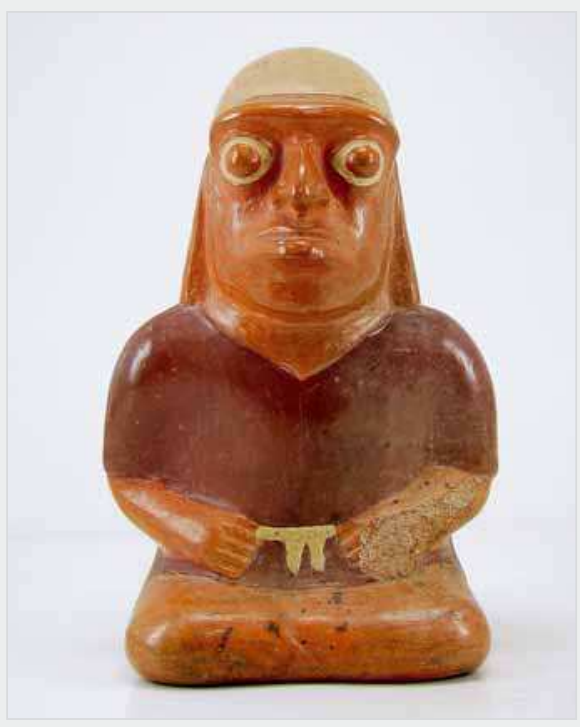

Abb. 3. Exophthalmus (\& Kretinismus?) (Larco H. N ML013642).

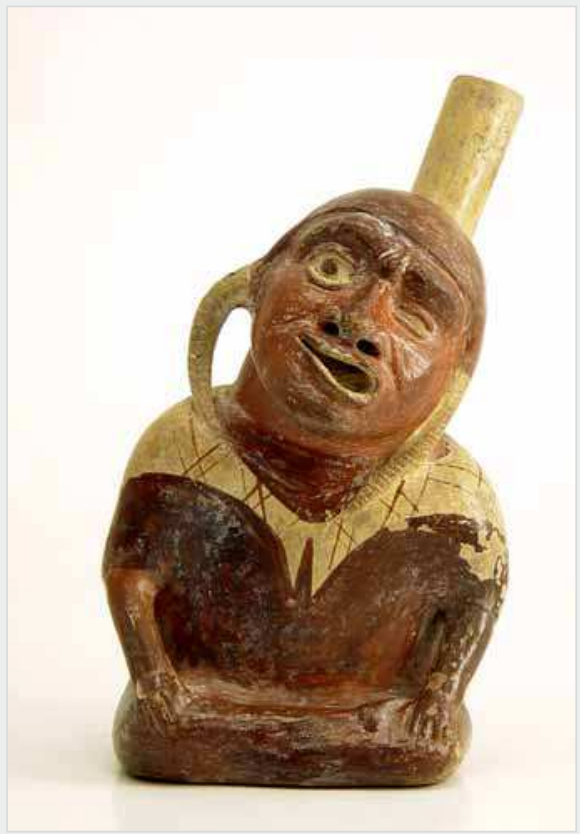

Abb. 5. Facialisparese (Larco H. N ML002737).
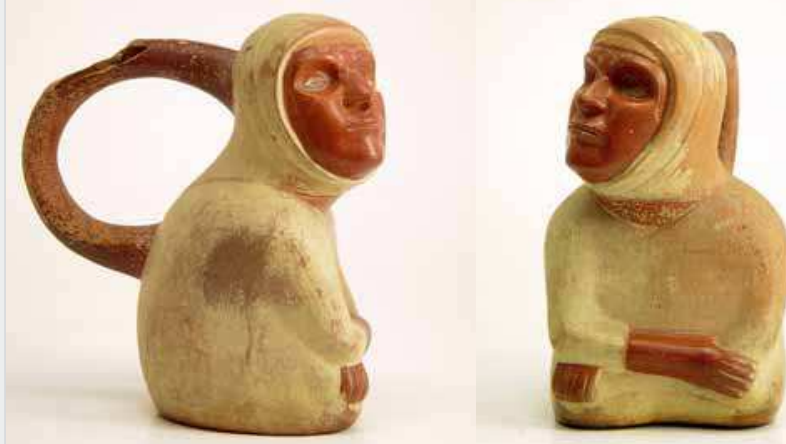

Abb. 7. Blinder (Larco H. No ML002821).

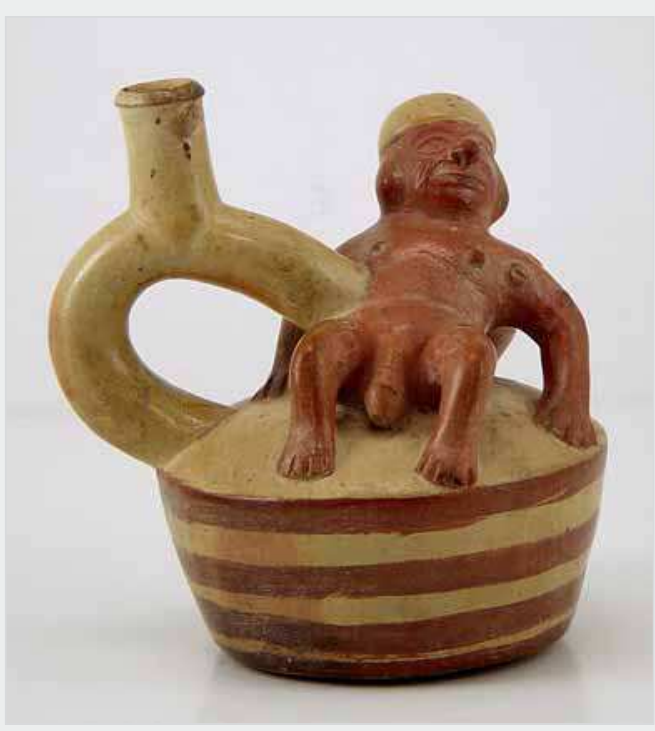

Abb. 4. Kutane und genitale Pusteln, deformierte Nase (wurde als Syphilis gedeutet, Larco H. No ML004299); diese war auch schon vor Kolumbus in der alten Welt auch vorhanden [43].

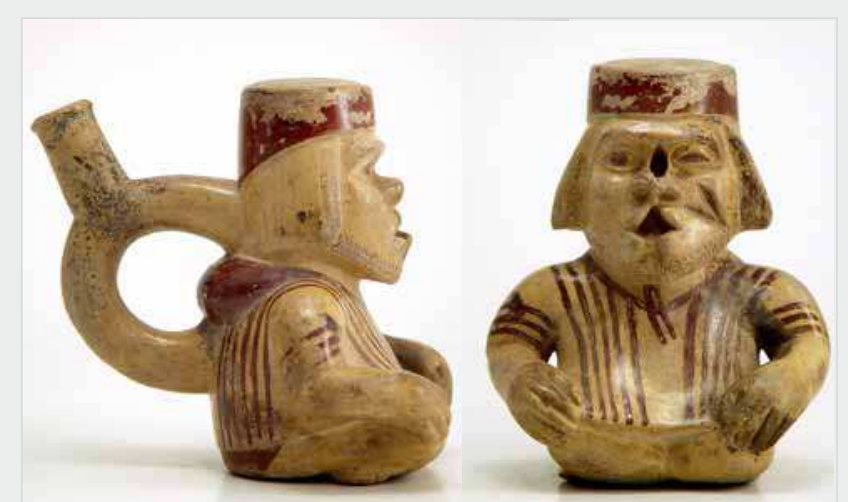

Abb. 6. Hasenscharte (Larco H. № ML001489).

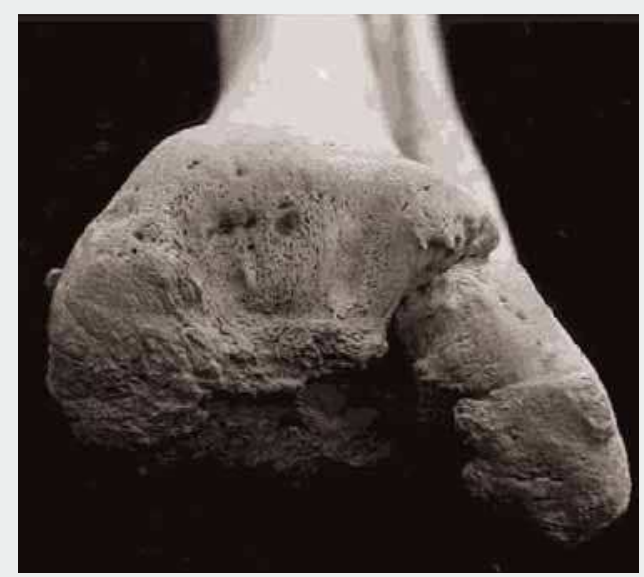

Abb. 8. Reaktive Knochenbildung nach Amputation; die hohe Knochendichte weist auf Belastung hin (aus VerANo et al., 2000). 


\section{Schlussfolgerung}

Die (Volks)medizin (Latein)amerikas stellt ein Kontinuum über mindestens drei Jahrtausende dar, wobei europäische und afrikanische Elemente - oft auch ,manu militari' - in den letzten Jahrhunderten integriert wurden. Am Beispiel von Kulturen in der Andenregion konnte gezeigt werden, wie vielfältig und heterogen die überlieferten und auch heute noch lebenden Elemente sind. Die Indios hatten schon vor der Conquista zahlreiche Krankheiten und Traumatismen; sie versuchten mit diesen so gut wie möglich und ihrer Weltanschauung entsprechend umzugehen. Wer sich näher mit der (Volks) medizin (Latein)Amerikas befassen will, sollte dies ohne ,a prioris' tun, denn nur so wird er der „Wahrheit“ näher kommen. Man wird kaum das verlorene Paradies auf Erden oder irgendwelche „Wunder“ antreffen, sicher aber interessante Gedankenanstösse.

\section{Danksagung und Angaben zur finanziellen Unterstützung}

An dieser Stelle möchten wir allen danken, die uns bei der Erstellung dieser Arbeit unterstützt haben. Unser ganz besonderer Dank geht an Frau Hannelore Deubel, NeukirchenVluyn, für die sorgfältige Durchsicht des Manuskripts.

Die Abbildungen 2-7 wurden freundlicherweise zur Verfügung gestellt von:

Museo Larco, Av. Bolívar 1515, Pueblo Libre Lima 21 - Perú

http://museolarco.perucultural.org.pe

Diese Arbeit erhielt keine finanzielle Unterstützung.

\section{Literatur}

1. WHO Traditional medicine. Fact sheet $\mathrm{N}^{\circ} 134$ Revised May 2003. http://www.who.int/ mediacentre/factsheets/ fs134/en/

2. Bodeker G, Ong CK, Grundy C, Burford G, Shein K. WHO Global Atlas of Traditional, Complementary and Alternative Medicine: Text Volume \& Map Volume Kobe Japan: WHO Centre for Health Development; 2005.

3. Heinrich $\mathrm{M}$ Ethnobotany and its role in drug development. Phytotherapy Research 2000: 14:479-488.

4. Rivara de Tuesta, ML. Wiracocha (Dios), pacha (mundo) y runa (hombre) en la cultura prehispánica (incaica) Zitiert in Pensamiento prehispánico y filosofía colonial en el Perú. Lima, FCE, Tomo I, 2000, pp. 98-132.

5. Dr. Roberto Campos Navarro ¿Existe el empacho en Cuba? Medisan, 2004;8(3):4-12.

6. Hill DM., Traditional medicine in contemporary contexts. Protecting and Respecting Indigenous Knowledge and Medicine National Aboriginal Health Organization (NAHO) March 19, 2003. http://www.naho.ca/english/ pdf/research_tradition.pdf

7. http://www.foodnews.ch/allerlei/20 historisches/Kartoffel_Geschichte.htm
8. Pardo OB. Estudio comparativo de ocho especies americanas de uso medicinal en Mozambique. Chloris chilensis 1999, 6. № 1 . http://www.chlorischile.cl/mozambique/INTR ODUCCION.htm

9. Sheldon PS, Kekwick RG, Sidebottom C, Smith CG, Slabas AR 3-Oxoacyl-(acyl-carrier protein) reductase from avocado (Persea americana) fruit mesocarp. Biochem J. 1990: 271:713-720.

10. Kawagishi $H$, Fukumoto $Y$, Hatakeyama $M$, He $P$, Arimoto $H$, Matsuzawa $T$, Arimoto $Y$, Suganuma $H$, Inakuma $T$, Sugiyama K Liver injury suppressing compounds from avocado (Persea americana).J Agric Food Chem. 2001;49:2215-2221.

11. Conde Garcia EA, Nascimento VT, Santiago Santos A.B. Inotropic effects of extracts of Psidium guajava L. (guava) leaves on the guinea pig atriumBraz J Med Biol Res 2003, 36:661-668.

12. Pieper B,Caliri MH Nontraditional wound care: A review of the evidence for the use of sugar, papaya/papain, and fatty acids. J Wound Ostomy Continence Nurs (2003 Jul) 30(4):175-83

13. Otuka ES,Pedrazzani ES,Pioto MP [The use of papain in plantar ulcers] Uso da papaina na ulcera plantar. Rev Bras Enferm (1996 Apr-Jun) 49(2):207-14

14. Ordi J, Alonso PL, de Zulueta J, Esteban J, Velasco M, Mas E, Campo E, Fernandez PL The severe gout of Holy Roman Emperor Charles V. N Engl J Med. 2006 Aug 3; 355 (5):516-20

15. Suzanne Austin Alchon The Great Killers in Precolumbian America, A Hemispheric Perspective LATIN AMERICAN POPULATION HISTORY BULLETIN, Fall 1997, Number 27, http://www.hist.umn.edu/ rmccaa/laphb/27f all97/laphb27a.htm

16. Drusini AG, Carrara N, Orefici G, Rippa Bonati M. Palaeodemography of the Nasca valley: reconstruction of the human ecology in the southern Peruvian coast. Homo (2001) 52(2): 157-72

17. Trinkaus E The Alto Salaverry child: a case of anemia from the Peruvian preceramic. Am J Phys Anthropol (1977 Jan) 46(1):25-8.

18. Elzay RP, Allison MJ, Pezzia A A comparative study on the dental health status of five precolumbian Peruvian cultures. Am J Phys Anthropol (1977 Jan) 46(1):135-9.

19. Allison, M.J., Pezzia, A., Hasegawa, L., and Gerszten, E: A Case of Hookworm Infestation in a Pre-Columbian American. - Amer. J. Phy. Anthrop1974., 41:103-105.

20. Ortega YR, Bonavia D Cryptosporidium, Giardia, and Cyclospora in ancient Peruvians. J Parasitol (2003 Jun) 89(3):635-6

21. Carod Artal FJ, Vazquez Cabrera CB [Neurological paleopathology in the pre-Columbian cultures of the coast and the Andean plateau (I). Artificial cranial deformation] Paleopatologia neurologica en las culturas precolombinas de la costa y altiplano andino (I). Deformaciones craneales intencionales. Rev Neurol (2004 Apr) 38(8):791-7.

22. Salo WL, Aufderheide AC, Buikstra J, Holcomb TA Identification of Mycobacterium tuberculosis DNA in a pre-Columbian Peruvian mummy. Proc Natl Acad Sci U S A (1994 Mar 15) 91(6):2091-4.

23. Urton G, Brezine CJ Khipu accounting in ancient Peru. Science (2005 Aug 12) 309 (5737):1065-7.

24. Verano JW Health and Medical Practices in the Pre-Columbian Americas Perspectives in health \& medicine 1999, Volume 4 - No.1. http://www.paho.org/English/DPI/Number7 article2.htm

25. Velarde OF. Fuentes históricas de la medicina prehispánica. Enfermedades del Aparato Digestivo OCTUBRE 2002;5:5-8.
26. Aparicio Mena AJ. Idea de salud para culturas riginarias mesoamericanas e idea de salud para los orientales. Revista de Antropología Experimental. 2004; 4:1-15. www.ujaen.es/huesped/rae

27. Report from the ICSU Study Group on Science and Traditional Knowledge. Science and Traditional Knowledge, March 2002, Section3.

28. Jorgensen JB Trepanation as a therapeutic measure in ancient (pre-Inka) Peru. Acta Neurochir (Wien) (1988) 93(1-2):3-5.

29. Rifkinson-Mann S Cranial surgery in ancient Peru. Neurosurgery (1988 Oct) 23(4):411-6.

30. Jaime Herrera Pontón La Medicina en la Época Precolombina Los Psicoestimulantes y los venenos de flechas: Precursores de la Anestesia. Revista MEDICINA. http://anm.encolombia.com/academ24360medicinaprecolombina.htm.

31. R. R. Griffiths \&W. A. Richards \& U. McCann \& R. Jesse Psilocybin can occasion mysticaltype experiences having substantial and sustained personal meaning and spiritual significance. Psychopharmacology, 2006, DOI 10. 1007/s00213-006-0457-5.

32. Zitate Blanco M. El Inca Garcilaso, un indio antártico (1539-1616). Estudios Públicos 2002, 86: 377-476.

33. Froemming $\mathrm{S}$ Traditional use of the Andean flicker (Colaptes rupicola) as a galactagogue in the Peruvian Andes. J Ethnobiol Ethnomedicine (2006), 2:23.

34. Springfield AC, Cartmell LW, Aufderheide AC, Buikstra J, Ho J. Cocaine and metabolites in the hair of ancient Peruvian coca leaf chewers. Forensic Sci Int (1993 Dec) 63(1-3):269-75

35. Sepúlveda JD. De la coca a la cocaina. Ars Medica 7,7:1-16.

http://escuela.med.puc.cl/publ/arsmedica/Ars Medica7/Art02.htm

36. Gryfe A. Pathology gone to pot: Paleopathology on Mochica ceramics The Canadian Journa of Plastic Surgery 2004, Volume 12, Number 2. http://www.pulsus.com/Plastics/02_04/gryf_ ed2.htm

37. Larco-Hoyle, R. Los Mochicas Tomo II. Lima 1938, pp. 226-265.

38. Serarcangeli C, Pennica A [Testimonies of an autochthonous illness on the anthropomorphic pottery in ancient Peru] Testimonianze di una malattia autoctona nella ceramica de Peru precolombiano. Med Secoli (1996) 8(1): 125-41.

39. Pirsig W [Aplasia and hypoplasia of the side of the nose in the Mocha Period and today Auris Nasus Larynx (1989) 16 Suppl 1:S47-52

40. Rick FM, Rocha GC, Dittmar K, Coimbra CE Reinhard K, Bouchet F, Ferreira LF, Araujo A Crab louse infestation in pre-Columbian America. J Parasitol (2002 Dec) 88(6):1266-7.

41. Verano JW, Anderson JS, Franco R. Foot Amputation by the Moche of Ancient Peru: Osteological Evidence and Archaeological Context Int. J. Osteoarchaeol. 2000,10:177-188.

42. F J Carod-Artal, C. B Vázquez Cabrera Malformaciones y parálisis faciales en la cerámica de las culturas precolombinas Moche y Lambayeque. Neurología 2006;20(6):297-303.

43. Meyer, C; Jung, C; Kohl, T; Poenicke, A Poppe, A; Alt, KW. Syphilis 2001-a palaeopathological reappraisal. Homo 2002;53(1): 39-58.

\section{Korrespondenzadresse:}

Prof. Dr. med. Reinhard Saller

UniversitätsSpital Zürich

Dep. für Innere Medizin

Institut für Naturheilkunde

Rämistrasse 100, CH-8091 Zürich

reinhard.saller@usz.ch 